\title{
Ethical Challenges in the Management of Pregnancies Complicated by Fetal Anomalies
}

\author{
${ }^{1}$ Frank A Chervenak, ${ }^{2}$ Laurence B McCullough, ${ }^{3}$ Daniel Skupski, ${ }^{4}$ Stephen T Chasen \\ ${ }^{1}$ The New York-Presbyterian Hospital, Weill Medical College of Cornell University, New York, USA \\ ${ }^{2}$ Center for Medical Ethics and Health Policy, Baylor College of Medicine, Houston, Texas, USA \\ ${ }^{3}$ The New York Hospital Queens, Weill Medical College of Cornell University, New York, USA \\ ${ }^{4}$ The New York-Presbyterian Hospital, Weill Medical College of Cornell University, New York, USA \\ Correspondence: Frank A Chervenak \\ The New York-Presbyterian Hospital, Weill Medical College of Cornell University \\ Department of Obstetrics and Gynecology, 525 East 68th Street - J130, New York, NY 10025 \\ Telephone: (212) 746-3045 FAX: (212) 746-8717
}

\begin{abstract}
Ethics is an essential dimension of the clinical management of pregnancies complicated by fetal anomalies. Appealing to the ethical principles of beneficence and respect for autonomy, this reviews explicates the ethical concept of the 'fetus as a patient'. This concept provides the basis for a comprehensive approach to ethical challenges in the management of pregnancies complicated by fetal anomalies. Practice, ethically justified guidance is given for the physician's role in counseling pregnant women about aggressive management, termination of pregnancy, selective termination of multifetal pregnancies, nonaggressive management, and cephalocentesis.
\end{abstract}

Key words: Ethics, ultrasound, abortion, fetal research, cephalocentesis, autonomy.

\section{Learning objectives}

1. To understand the basic ethical principles of beneficence and respect for autonomy.

2. To appreciate different senses of the fetus as a patient.

3. To apply basic ethical principles and the concept of the fetus as a patient to the management of fetal anomalies diagnosed by ultrasound before and after fetal viability.

\section{INTRODUCTION}

The management of pregnancies complicated by fetal anomalies confronts physicians specializing in obstetric ultrasound with ethical challenges. Based on our previous work, we provide an ethical framework within which responsible decision making about these challenges can be accomplished and implemented in clinical practice. ${ }^{1}$ We first provide introduction to medical ethics and its two basic principles, i.e. beneficence and respect for autonomy. We then use these ethical principles to structure the important concept of the fetus as a patient. We then address management options before and after viability.

\section{BASIC ETHICAL PRINCIPLES}

Medical ethics begins with the physician's basic obligation to protect and promote the health-related interests of patients. In order to make this general obligation clinically applicable, we interpret it in terms of two perspectives on the interests of patients, that of the physician and that of the patient. ${ }^{2,3}$

\section{Beneficence}

The ethical principle of beneficence translates into medicine's expert, evidence-based perspective on the health-related interests of the patient into clinical decision making and practice. Beneficence obligates the obstetrician to seek the greater balance of clinical benefits over clinical harms for the patient in patient care. Relying on rigorous clinical judgment, informed by the best available evidence and a sustained commitment to excellence in clinical practice, the obstetrician should identify clinical strategies that are reliably expected to result in the greater balance of clinical benefits, i.e. the protection and promotion of health-related interests, over clinical harms, i.e. risks to those interests. Beneficence has a long history in the global history of medical ethics. In Western medical ethics, for example, it dates back at least to Oath and accompanying texts of Hippocrates. ${ }^{4}$ The Oath requires physicians to act in a manner that will "benefit the sick according to my ability and judgment."

Beneficence in obstetrics should not be confused with the principle of nonmaleficence, which is also known as primum non-nocere or "first, do no harm". Primum non nocere appears neither in the Hippocratic Oath nor in the texts that accompany the Oath; rather, the principle of beneficence was the primary consideration of the Hippocratic writers. In epidemics, the text reads, "As to diseases, make a habit of two things - to help or 
to at least do no harm". 5 Indeed, the historical origins of primum non-nocere remain obscure. This seemingly arcane point is not merely historical but it is conceptual and clinical: if primum non-nocere were to be made the primary ethical principle of obstetrics, then virtually all invasive aspects of obstetrics would be unethical because of the risks they involve for the pregnant woman. Given the psychosocial risks of ultrasound examination, it, too, might have to be judged unethical from the perspective of "first do no harm".

\section{Respect for Autonomy}

An evidence-based clinical perspective on the patient's healthrelated and other interests is not the only legitimate perspective. The patient has her own perspective on her health-related and other interests that must be taken into consideration by the physician. ${ }^{2}$ The pregnant patient has developed a set of values and beliefs and, as an adult, she should be presumed to be capable of making judgments about what will and will not protect and promote her health-related and other interests that she regards as important. This presumption guides clinical judgment and decision making of the physician, unless there is reliable evidence of significant and irreversible clinical deficits in her decision making processes. We emphasize that pregnant women may appeal to values and beliefs that go beyond their health-related interests, e.g. religious beliefs or beliefs about how many children are desirable to have. Beneficence-based clinical judgment is limited by the competencies of medicine, and therefore, does not provide the physician with the intellectual or moral authority to assess the worth or meaning to the pregnant woman of her own non-health-related interests. Such are matters solely for the pregnant woman to determine for herself.

The patient's perspective on her health-related and other important interests is translated into clinical practice in the ethical principle of respect for autonomy. Respect for autonomy obligates the physician to respect the integrity of the patient's values and beliefs, to respect her perspective on her interests, and to implement clinical strategies authorized by her as the result of the informed consent process. The informed process has three elements: (i) disclosure by the physician to the patient of adequate information about the patient's condition and medically reasonable alternatives for its management (i.e. alternatives supported in beneficence-based clinical judgment); (ii) understanding of that information by the patient; and (iii) a voluntary decision by the patient to authorize or refuse proposed management. ${ }^{2,6}$

\section{ETHICAL CONCEPT OF THE FETUS AS A PATIENT}

The obstetrician's commitment to protect and promote the pregnant patient's health-related interests creates beneficence- based obligations to her. The woman's own perspective on her interests and the physician's commitment to respect her values and preferences create the physician's autonomy-based obligations. The fetus cannot meaningfully be said to possess values and beliefs, because of its insufficiently developed central nervous system. There is, therefore, no valid basis for claiming that a fetus has a perspective on its interests. There can, therefore, be no autonomy-based obligations of the physician to any fetus. ${ }^{2}$ Nonetheless, the obstetrician does have a perspective on the fetus's health-related interests, and therefore, can have beneficence-based obligations to the fetus, but only when the fetus is a patient. The ethical concept of the fetus as patient requires detailed elaboration, a task to which we now turn.

An important aspect of the concept of the fetus as a patient is that the concepts of fetal rights or fetal personhood have no meaning and no clinical application to the fetus in obstetric ethics. Current controversies about "right to life," a concept with limited application to patients from non-Western cultures, can be avoided in responsible decision making about the clinical management of pregnancies complicated by fetal anomalies.

Two of the authors (FAC and LBM) have argued that beneficence-based obligations to the fetus exist when the fetus can later, after birth, achieve independent moral status. ${ }^{2}$ For the fetus, to be regarded as a patient, two conditions must be met: (i) the fetus is presented to the physician; and (ii) there exist medical interventions, whether diagnostic or therapeutic, that reliably can be expected to result in a greater balance of clinical goods over clinical harms for the fetus in its future. The clinical application of the concept of the fetus as a patient depends on links to its later becoming a child and, later still, achieving independent moral status.

\section{Viable Fetus as a Patient}

One link to becoming a patient is viability. Viability should not be understood as an exclusively biological property of the fetus but also in terms of technological factors. By virtue of both factors, a viable fetus can exist ex utero, and subsequently, become a child and later achieve independent moral status. In countries with different levels of technological capacity, viability is a close correlate of access to technological capacity. When access to technology is present, viability occurs at approximately the end of 24 weeks of gestational age. ${ }^{7}$

\section{Previable Fetus as a Patient}

The only possible link, between the previable fetus and the child it can become, is the pregnant woman's autonomy. Technological factors cannot result in the previable fetus becoming a child: this is simply what previable means. When the fetus is previable, the link between a fetus and the child it can become, can be established only by the pregnant woman's 
decision to confer the status of being a patient on her previable fetus. The previable fetus has no claim to the status of being a patient independently of the pregnant woman's autonomy. The pregnant woman is, therefore, free to withhold, confer, or having once conferred, withdraw the status of being a patient on or from her previable fetus according to her own values and beliefs.

\section{MANAGEMENT OPTIONS BEFORE AND AFTER VIABILITY}

\section{Before Viability}

\section{Termination of Pregnancy}

Before viability, the clinical management of a pregnancy complicated by fetal anomalies is ethically straightforward. The pregnant woman is free to withhold or withdraw the moral status of being a patient from any previable fetus, including the fetus diagnosed by ultrasound examination to have anomalies. Counseling by the physician should be rigorously nondirective. That is, the physician should offer medically reasonable alternatives, but should not make any recommendations for or against any particular alternative. The woman should be given the choice between abortion and continuing her pregnancy to viability and thus toward term, without being influenced by statements of the physician's personal views about rearing a child with such an anomaly or about abortion. If the woman elects to terminate her pregnancy, termination should be performed unless the physician has moral objections to abortion in individual conscience, which should be respected by the patient and the physician's colleagues. As a matter of professional conscience, all physicians, including those unwilling to perform abortions on the grounds of individual conscience, should make an appropriate referral. If the woman elects to continue her pregnancy, she should be apprised about decisions that will need to be made later, so that she can begin to plan the rearing of her child. ${ }^{1,2}$

Respect for autonomy requires that the physician should not judge the reasons a woman has for terminating a previable pregnancy. Respect for autonomy also requires the physician to be alert to substantially controlling or even coercive influences on her decisions about the clinical management of a pregnancy, such as from her husband, partner, or potential grandparents. The physician should therefore advocate for her preferences, whatever they may be, in order to protect her from such substantial control and coercion. ${ }^{2}$

\section{Selective Termination of Multifetal Pregnancies}

An important subset of the option of termination of pregnancies is fetal reduction and selective termination of multiple pregnancies. ${ }^{8-10}$ The three ethically justifiable indications for reduction or selective termination of multiple pregnancies are related to three possible goals for a multiple pregnancy: (i) a pregnancy that results in the live birth of one or more infants with minimal neonatal morbidity and mortality; (ii) a pregnancy that results in the live birth of one or more infants without anomalies detected antenatally; and (iii) a pregnancy that results in a singleton live birth. ${ }^{9}$

1. First indication: Achieving a pregnancy that results in live birth with minimal neonatal morbidity and mortality.

In triplet or higher order pregnancies, while the goal of live birth with minimal neonatal mortality and morbidity is more than remotely possible, there are significant increased risks of both fetal morbidity and mortality, depending on the number of fetuses when there are four or more. Fetal reduction either makes it possible to achieve, or increases the likelihood of achieving, the goal of live birth of infant(s) with minimal neonatal morbidity and mortality. ${ }^{8}$ The first indication applies to cases in which the woman's goal is to maximize the probability of live birth. In current clinical judgment, this is best achieved by having two fetuses remain after the procedure has been performed.

It may at first appear that this indication for fetal reduction is ethically unjustified because it violates beneficence-based obligations to the fetus as patient. On closer examination, this is not the case, because the moral status of being a patient is conferred on the previable fetus only as a function of the pregnant woman's decision to do so. The clinical reality is that, for pregnancies in this category, the pregnant woman's decision to confer such status on all of the fetuses will jeopardize all of the fetuses. For some of the fetuses to become patients, the moral status of being a patient must be withheld from others. Thus, fetal reduction does not involve the killing of patients and is, therefore, justified in obstetric ethics.

2. Second indication: Achieving a pregnancy that results in live birth without antenatally detected anomalies.

The goal of obstetric care in this cases differs from that of the first indication: live birth(s) without antenatally detected fetal anomalies. When a woman elects to selectively terminate a fetus with a detected anomaly, she, in effect, with holds from that fetus the moral status of becoming a patient and thus cannot reasonably be thought to be violating, in any way, beneficencebased obligations to that fetus. Nor can the physician who performs the procedure. The remaining fetus(es) will be taken to term and thus have conferred on it, or them, by the pregnant woman the moral status of being a patient(s). The possible risks of increased morbidity and mortality for remaining fetuses must be evaluated in the particular context of whether the anomaly is of such severity to justify possible compromise of the beneficence-based obligations to the remaining fetus(es). At the present time, risks of the selective termination procedure to the survivor fetus(es) are so infrequent that one cannot justify over-riding beneficence-based obligations to remaining fetuses not to perform the procedure. ${ }^{10}$ 
3. Third indication: Achieving a pregnancy that results in a singleton live birth.

These cases involve the pregnant woman's decision to have a single child rather than more than one child from her pregnancy. The woman with holds the moral status of being a patient from one or more of the fetuses, something she is free to do as a matter of exercising her autonomy to set her own goals for her pregnancy, as explained above. The pregnant woman also confers the status of being a patient on the fetus that survives reduction to the singleton, i.e. the one that she intends to take to term. As a consequence, there are beneficence-based obligations on her part and her physician's part to the singleton fetus to avoid significant harm that might result from the reduction. Clinical judgment at this time does not support the contention that harm will occur with high probability. ${ }^{10}$

\section{Individual Conscience and Selective Termination of Multiple Pregnancies}

The clinical strategy for implementing the decisions about the disposition of pregnancies complicated by fetal anomalies is the informed consent process. As the outcome of the process, the pregnant woman may make a decision that the physician cannot accept as a matter of individual conscience as distinct from professional conscience. ${ }^{2}$ There will also be a wide spectrum among physicians of the acceptability in individual conscience of fetal reduction or selective termination, just as there is concerning the acceptability of abortion.

It is well accepted in medical ethics that the exercise of autonomy by a patient cannot justifiably oblige a physician to act in a way that is unacceptable as a matter of individual conscience, with the exception of emergencies. ${ }^{2}$ This exception does not apply to elective abortion. It follows that a physician legitimately exercises his or her own autonomy when it is found that this sort of moral incompatibility exists between himself/ herself and his/her patient.

\section{After Viability}

After viability, aggressive management is the ethical standard of care: optimizing perinatal outcome by utilizing effective antepartum and intrapartum diagnostic and therapeutic modalities. We will discuss three other management options, i.e. termination of pregnancy, non-aggressive management, and cephalocentesis. These options are ethically challenging and best avoided through early diagnosis. ${ }^{1,2}$

\section{Termination of Pregnancy}

One management option is termination of pregnancy after fetal viability. This option is ethically acceptable when there is (i) certainty of diagnosis, and either (iia) certainty of death as an outcome of the anomaly diagnosed, or (iib) in some cases of short-term survival, certainty of the absence of cognitive developmental capacity as an outcome of the anomaly diagnosed. ${ }^{2,11}$ When these criteria are satisfied, recommending a choice between non-aggressive management and termination of pregnancy is justified. Anencephaly is a classic example of a fetal anomaly that satisfies these criteria. ${ }^{11}$

An argument can also be made that other anomalies, such as trisomy 13 , trisomy 18 , renal agenesis, thanatophoric dysplasia, alobar holoprosencephaly and hydranencephaly, should count as anomalies that could ethically justify third trimester abortion. ${ }^{12}$ With these anomalies, either death is already a certain or a near certain outcome or the certain or near certain absence of cognitive developmental capacity is tantamount to death and so in beneficence-based clinical judgment causing death is an acceptable outcome.

For many anomalies, such as Down syndrome, spina bifida, isolated hydrocephalus, diaphragmatic hernia, achondroplasia, and most cardiac anomalies, neither death nor absence of cognitive developmental capacity can reliably be regarded as a certain or near certain outcome. While these anomalies do involve incremental risks of mental and physical morbidity and mortality, they do not justify third trimester abortion: rigorous clinical evaluation rules out regarding these conditions as tantamount to death or the absence of cognitive developmental capacity. For such anomalies, the beneficence-based prohibition against terminating the life of a viable fetus remains robustly intact. Any clinical judgment that does not address and defeat this beneficence-based prohibition is defective on ethical grounds, and therefore, is inconsistent with the professional integrity. ${ }^{12,13}$

The logic of beneficence-based obligations to the fetal patient means that the woman's beneficence-based obligations are the same as those of her physician. She should act on those obligations provided that the risks to her of doing so are reasonable. ${ }^{2}$ The risks of continuing a viable pregnancy to term are in almost all cases reasonable. In the rare instance in which the woman's health necessitates delivery, all efforts should be made to help the child. The pregnant woman's autonomy should, therefore, be understood by the woman and her physician to be constrained by the beneficence-based prohibition against killing the third trimester fetal patient in these cases. Thus, a woman's exercise of autonomy to request a third trimester abortion for a fetus with an anomaly such as Down syndrome, lacks ethical authority. ${ }^{2}$ Therefore, as a matter of professional integrity, no physician should carry out such a request.

\section{Non-aggressive Obstetric Management}

A second management option is non-aggressive obstetric management. This management option is ethically acceptable when there is (i) a very high probability, but sometimes less 
than complete certainty, about the diagnosis and, either (iia) a very high probability of death as an outcome of the anomaly diagnosed, or (iib) survival with a very high probability of severe and irreversible deficit of cognitive developmental capacity as a result of the anomaly diagnosed. ${ }^{2,14}$ When these two criteria apply, a choice between aggressive or non-aggressive management should be offered. Encephalocele is a classic example of a fetal anomaly that satisfies these criteria.

\section{Cephalocentesis for Intrapartum Management of Hydrocephalus}

Cephalocentesis involves the drainage of an enlarged fetal head, secondary to hydrocephalus. ${ }^{15,16}$ Cephalocentesis should be performed under simultaneous ultrasound guidance so that needle placement into the cerebrospinal fluid is facilitated. Cephalocentesis is a potentially destructive procedure. Perinatal death following cephalocentesis has been reported in over $90 \%$ of cases. ${ }^{17}$ If decompression is performed in a controlled manner, mortality may be reduced.

Isolated fetal hydrocephalus: There is considerable potential for normal, sometimes superior, intellectual function for fetuses with even extreme, isolated hydrocephalus. ${ }^{18-20}$ However, infants with isolated hydrocephalus experience a greater incidence of mental retardation and early death than the general population. A viable at-term fetus with isolated hydrocephalus is a fetal patient, because neither of the two exceptions described above (certainty of diagnosis and certainty of outcome) apply. ${ }^{2}$

The beneficence-based obligation of the physician caring for the fetus with macrocephaly is to recommend strongly, and to obtain the woman's informed consent to perform, cesarean delivery. This clinical intervention involves the least risk of mortality, morbidity, and disability for the fetus compared with cephalocentesis to permit subsequent vaginal delivery. Even when performed under maximal therapeutic conditions (i.e. under sonographic guidance), cephalocentesis cannot reasonably be regarded as protecting or promoting the health-related interests of the fetal patient with isolated hydrocephalus with macrocephaly.

Hydrocephalus with severe associated abnormalities: Some abnormalities that occur in association with fetal hydrocephalus are severe in nature. "Severe" abnormalities are those that either are (i) incompatible with continued existence, e.g. bilateral renal agenesis or thanatophoric dysplasia with cloverleaf skull, (ii) compatible with survival in some cases but result in virtual absence of cognitive function, e.g. trisomy 18 or alobar holoprosencephaly. ${ }^{17,21}$ Because there is no available intervention to prevent postnatal death in the first group, there is no beneficence-based obligation of the physician and the pregnant woman to attempt to prolong the life of the fetal patient. For the second group, the beneficence-based obligation of the physician and the pregnant woman to sustain the life of the fetal patient is minimal because the disability imposed by the abnormality is severe. In these cases the potential for cognitive development is virtually absent. Such fetuses are fetal patients to which the physician and pregnant woman have only a minimal beneficence-based obligation.

In these cases, the pregnant woman is released from her beneficence-based obligation to the at-term fetal patient to place herself at risk, because no significant good can be achieved by cesarean delivery for the fetal patient or the child it will become. The autonomy-based and beneficence-based obligations of the physician to the pregnant woman are the only ethically relevant considerations. We conclude that the physician's over-riding moral obligations are to the pregnant woman's voluntary and informed decision about employment of cephalocentesis.

Hydrocephalus with other associated anomalies: Between the extreme cases of isolated hydrocephalus and hydrocephalus with severe associated abnormalities are cases of hydrocephalus associated with macrocephaly with other abnormalities with varying degrees of impairment of cognitive physical function. These range from hypoplastic distal phalanges to spina bifida to encephalocele. ${ }^{17,21}$ Because these conditions have varying prognoses, it would be clinically inappropriate, and therefore, ethically misleading to treat this third category as homogeneous. We, therefore, propose a distinction between different kinds of prognoses. The first we call "probably promising": there is a significant possibility the child will experience cognitive development with learning and physical disabilities that perhaps can be ameliorated to some extent. The second we call "probably poor": there is only a limited possibility for cognitive development because of learning and physical disabilities that cannot be ameliorated to a significant extent.

When the prognosis is probably promising, e.g. isolated arachnoid cyst, there are serious beneficence-based obligations to the fetal patient. However, they are not necessarily on the same order as those that occur in cases of isolated hydrocephalus (It has been suggested that any associated anomaly may increase the possibility of a poor outcome ${ }^{17}$ ). In such cases, the physician should recommend cesarean delivery, although perhaps not as strongly as in cases of isolated hydrocephalus. A pregnant woman's informed refusal of cesarean delivery should be respected.

In cases when the prognosis, even though uncertain, is probably poor, e.g. encephalocele, beneficence-based obligations to the fetal patient are less weighty than those owed to the fetal patient with a promising prognosis. These cases resemble those of hydrocephalus with severe anomalies, with the proviso that some, albeit limited, benefits can be achieved for the fetal patient by cesarean delivery and aggressive perinatal treatment. Nonetheless, the physician may in these cases 
justifiably accept an informed voluntary decision by the woman for cephalocentesis followed by vaginal delivery. However, the physician should not assume an advocacy role for such a decision with the same level of ethical confidence that he or she can in cases of hydrocephalus associated with severe anomalies.

\section{CONCLUSIONS}

Ethics is an essential dimension of the management of pregnancies complicated by fetal anomalies. The ethical concept of the fetus as a patient should guide the obstetrician in reaching ethically justified judgments about how to balance autonomybased and beneficence-based obligations to the pregnant woman and beneficence-based obligations to the fetus. These ethical obligations can be stratified in a clinically useful fashion into management of pregnancies before viability and management of pregnancies after viability. For previable pregnancies, respect for the pregnant woman's autonomy is the decisive ethical consideration, including the subset of selective termination for multifetal pregnancies. For viable pregnancies, beneficence-based obligations to the fetal patient support aggressive obstetric management with the well-defined exceptions of termination of pregnancy, non-aggressive management, and cephalocentesis.

\section{REFERENCES}

1. Chervenak FA, McCullough LB, Skupski D, Chasen DT. Ethical issues in the management of pregnancies complicated by fetal anomalies. Obstet Gynecol Surv 2003;58:473-83.

2. McCullough LB, Chervenak FA. Ethics in Obstetrics and Gynecology. Oxford University Press: New York, 1994.

3. Beauchamp TL, Childress JF. Principles of Biomedical ethics (5th edn). Oxford University Press: New York, 2000.

4. Temkin O, Temkin CL, Edelstein L. Ancient Medicine, Johns Hopkins University Press: Baltimore, 1967; p.3.

5. Hippocrates. Epidemics i:xi. WHS Jones, trans. Loeb Classical Library. Harvard University Press: Cambridge, 1923; vol 147.

6. Faden RR, Beauchamp TL. A History and Theory of Informed Consent. Oxford University Press: New York, 1986.
7. Chervenak FA, McCullough LB, Levene ML. An ethically justified, clinically comprehensive approach to periviability: gynaecologic, obstetric, perinatal, and neonatal dimensions. Br J Obstet Gynecol (in press).

8. Evans MI, Johnson MP, Quintero RA, Fletcher JC. Ethical issues surrounding multifetal pregnancy reduction and selective termination. Clin Perinat 1996;23:437-51.

9. Chervenak FA, McCullough LB, Wapner R. Three ethically justified indications for selective termination in multifetal pregnancy: a practical and comprehensive management strategy. J Assist Reprod Genet 1995;12:531-6.

10. Evans MI, Dommergues M, Wapner R, et al. International collaborative multifetal pregnancy indication: collaborative experience among the world's largest centers. Obstet Gynecol 1993;82:61-7.

11. Chervenak FA, Farley MA, Walters L, et al. When is termination of pregnancy during the third trimester morally justifiable? $\mathrm{N}$ Engl J Med 1984;310:501-4.

12. Chervenak FA, McCullough LB, Campbell S. Is third trimester abortion justified? Br J Obstet Gynæcol 1995; 102:434-5.

13. Chervenak FA, McCullough LB, Campbell S. Third trimester abortion: is compassion enough? Br J Obstet Gynaecol 1999;106:293-6.

14. Chervenak FA, McCullough LB. Nonaggressive obstetric management: an option for some fetal anomalies during the third trimester. JAMA 1989;261:3439-40.

15. Chervenak FA, Romero R. Is there a role for fetal cephalocentesis in modern obstetrics? Am J Perinatol 1984;1:170-3.

16. Chasen S, Chervenak FA, McCullough LB. The role of cephalocentesis in modern obstetrics. Am J Obstet Gynecol 2001;185:734-6.

17. Chervenak FA, Berkowitz RL, Tortora M, et al. Management of fetal hydrocephalus. Am J Obstet Gynecol 1985;151:933-7.

18. Raimondi AJ, Soare P. Intellectual development in shunted hydrocephalic children. Am J Dis Child 1974;127:664.

19. McCullough DC, Balzer-Martin LA. Current prognosis in overt neonatal hydrocephalus. J Neurosurg 1982;57:378.

20. Sutton LN, Bruce DA, Schut L. Hydranencephaly versus maximal hydrocephalus: an important clinical distinction. Neurosurgery 1980;6:35.

21. Nyberg DA, Mack LA, Hirsch J, et al. Fetal hydrocephalus: sonographic detection and clinical significance of associated anomalies. Radiology 1987;163:187. 